\title{
Formation documentaire au collégial : une enquête sur les pratiques
}

\author{
Library Training at the College Level: An Investigation into the \\ Practices
}

\section{Formación documental en el colegiado: encuesta sobre las prácticas}

\section{Anne-Frédérique Champoux}

Volume 62, numéro 1, janvier-mars 2016

URI : https://id.erudit.org/iderudit/1035927ar

DOI : https://doi.org/10.7202/1035927ar

Aller au sommaire du numéro

\section{Éditeur(s)}

Association pour l'avancement des sciences et des techniques de la documentation (ASTED)

\section{ISSN}

0315-2340 (imprimé)

2291-8949 (numérique)

\section{Découvrir la revue}

\section{Citer cet article}

Champoux, A.-F. (2016). Formation documentaire au collégial : une enquête sur les pratiques. Documentation et bibliothèques, 62(1), 33-40.

https://doi.org/10.7202/1035927ar

\section{Résumé de l'article}

En avril 2014, un comité de travail du Regroupement des bibliothèques collégiales du Québec (REBICQ) mène une enquête afin de dresser un portrait de l'offre de formation documentaire dans le réseau des cégeps et collèges. L’objectif est de recueillir des données actualisées sur les pratiques, les modalités et les contenus des formations proposées aux étudiants du niveau collégial. Les résultats obtenus montrent que la formation documentaire revêt une grande importance dans les bibliothèques du REBICQ ayant répondu à l'enquête, car 100\% d'entre elles en proposent. L'étude révèle par ailleurs que les contenus présentés en formation sont pertinents et diversifiés. On constate également que les ateliers en présentiel, s'insérant dans une multitude de cours et programmes, rejoignent chaque année un pourcentage important d'étudiants, soit près de $40 \%$ des cégépiens inscrits à l'enseignement régulier.
Tous droits réservés (C) Association pour l'avancement des sciences et des techniques de la documentation (ASTED), 2016
Ce document est protégé par la loi sur le droit d'auteur. L’utilisation des services d'Érudit (y compris la reproduction) est assujettie à sa politique d'utilisation que vous pouvez consulter en ligne.

https://apropos.erudit.org/fr/usagers/politique-dutilisation/ 


\title{
Formation documentaire au collégial : une enquête sur les pratiques
}

\author{
ANNE-FRÉDÉRIQUE CHAMPOUX \\ Spécialiste en moyens et techniques d'enseignement, Cégep du Vieux Montréal \\ Animatrice du Regroupement des bibliothèques collégiales du Québec (2015-2016) \\ afchampoux@cvm.qc.ca
}

\section{RÉSUmé | ABSTRACT | RESUMEN}

En avril 2014, un comité de travail du Regroupement des bibliothèques collégiales du Québec (REBICQ) mène une enquête afin de dresser un portrait de l'offre de formation documentaire dans le réseau des cégeps et collèges. L'objectif est de recueillir des données actualisées sur les pratiques, les modalités et les contenus des formations proposées aux étudiants du niveau collégial. Les résultats obtenus montrent que la formation documentaire revêt une grande importance dans les bibliothèques du REBICQ ayant répondu à l'enquête, car $100 \%$ d'entre elles en proposent. L'étude révèle par ailleurs que les contenus présentés en formation sont pertinents et diversifiés. On constate également que les ateliers en présentiel, s'insérant dans une multitude de cours et programmes, rejoignent chaque année un pourcentage important d'étudiants, soit près de $40 \%$ des cégépiens inscrits à l'enseignement régulier.

\section{Library Training at the College Level: An Investigation into the Practices}

In April 2014, a working committee, struck by the Regroupement des bibliothèques collégiales du Québec (REBICQ), undertook an investigation of the state of library training in cegeps and colleges. The purpose was to collect current data on the practices, the modalities and the content of the training sessions for college-level students. The results indicate that library training is very important to the libraries affiliated with the REBICQ who participated in the study; $100 \%$ of libraries offer such training sessions. The study also revealed that the content of these sessions is relevant and varied. It was also noticed that in-class sessions, as part of courses and programmes, reach about $40 \%$ of the students enrolled in regular programmes.

\section{Formación documental en el colegiado: encuesta sobre las prácticas}

En abril de 2014, un comité de trabajo del Regroupement des bibliothèques collégiales du Québec (REBICQ, Agrupación de bibliotecas colegiadas de Quebec) lleva a cabo una encuesta a fin de esbozar un retrato de la oferta de formación documental en la red educativa (cégep, colegios). El objetivo es recopilar datos actualizados acerca de las prácticas, las modalidades y los contenidos de las formaciones propuestas a los estudiantes del nivel colegial. Los resultados obtenidos muestran que la formación documental cuenta con una gran importancia en las bibliotecas del REBICQ que respondieron a la encuesta, ya que todas ellas proponen este tipo de formación. Asimismo, el estudio revela que los contenidos presentados son pertinentes $y$ diversificados. Constatamos igualmente que los talleres presenciales, que se insertan dentro de una multitud de cursos y programas, reúnen cada año a un alto porcentaje de estudiantes, aproximadamente un $40 \%$ de los inscriptos en la enseñanza regular de los cégep.

\section{Introduction}

I e Regroupement des bibliothèques collégiales (REBICQ), dont les activités ont débuté en 2012, a pour mission "de soutenir la mise en commun et le développement des expertises, des outils et des pratiques documentaires des bibliothèques collégiales" (REBICQa 2015). Pour mieux s'acquitter de son rôle, le REBICQ s'est doté de comités de travail qui s'attachent chacun à une problématique d'intérêt pour le réseau. C'est ainsi que le comité sur la formation documentaire a été formé. Le mandat donné à ce comité est de "réfléchi[r aux] facteurs qui font de la formation documentaire un soutien à la réussite" (REBICQb 2015). Avant de s'attaquer à cette question, les membres du comité ont jugé qu'il convenait d'abord d'en apprendre davantage sur les pratiques actuelles de formation, telles que mises en œuvre dans les différents cégeps et collèges affiliés au REBICQ. Le comité a alors inscrit à son plan de travail pour l'année 2013-2014 l'objectif de dresser un portrait de l'offre de formation documentaire au sein du réseau collégial en réalisant un questionnaire.

L'article qui suit présentera donc l'enquête réalisée en 2014 par le comité sur la formation documentaire en vue d'atteindre cet objectif. La méthodologie, les résultats obtenus, des recommandations et une ouverture sur les suites de l'enquête seront abordés.

\section{Revue de littérature}

L'enquête de 2014 est destinée à combler un vide, car il n'existe pas de données récentes sur les pratiques, les modalités et les contenus des formations proposées aux cégépiens, bien que divers articles aient abordé l'un ou l'autre de ces aspects par le passé. Dès 1977, un comité relevant de la Commission des directeurs de bibliothèques de la Fédération des cégeps s'est intéressé aux questions de "l'initiation des étudiants au travail personnel et [de] l'utilisation des ressources de la bibliothèque " (Lemaire 1978, 38). Déjà à l'époque, on constate que $82,5 \%$ des institutions proposent une initiation à la bibliothèque, le plus souvent sous la forme d'une visite guidée ( 30 répondants sur 40 ), ce qui correspond bien à la tendance du library orientation, prédominante dans les années 1970 (Bopp et Smith 2011). Lemaire en 
conclut que «les collèges sont sensibilisés à l'importance $d$ '[offrir] une initiation» destinée à faire connaittre les lieux, les ressources ou les services de la bibliothèque aux étudiants. Il déplore toutefois que seuls quatre des 40 cégeps sondés proposent des "guides de méthodologie de la recherche" (Lemaire 1978, 39). Selon l'auteur, " il revient aux bibliothécaires des cégeps d'enseigner aux étudiants comment la bibliothèque s'insère dans leur processus d'apprentissage, comment effectuer une recherche documentaire » (Lemaire 1978, 41), une tâche que peu de professionnels remplissent à ce moment.

Dix ans plus tard, Pagé et Reid (1988) se penchent sur l'évolution des méthodes employées en formation documentaire en conduisant une nouvelle enquête. Cette dernière est restreinte à 12 collèges de la région de Montréal; 9 répondants complètent le questionnaire. Pagé et Reid distinguent deux niveaux de formation documentaire : l'orientation et l'instruction. Selon leur catégorisation, la communication aux étudiants d'informations ayant trait " [aux] lieux, [aux] services, [aux] outils documentaires, [...] aux règlements, procédures et horaires " relève de l'orientation, tandis que celle portant "sur la méthodologie de recherche et sur l'utilisation des différents outils documentaires " participe de l'instruction (Pagé \& Reid 1988, 139). Les deux chercheuses remarquent que les méthodes de formation se rapportant à l'orientation sont encore les plus largement utilisées (100\% des institutions sondées offrent des visites de la bibliothèque), mais elles observent aussi que "cinq collèges sur neuf initient les étudiants à la méthodologie de recherche» (Pagé \& Reid 1988, 141), un changement notable par rapport aux constats émis à la suite de l'enquête précédente. Elles soulignent par ailleurs que toutes les bibliothèques consultées offrent un service d'aide individuelle aux étudiants, qu'on peut assimiler à un type de formation ad hoc. Il faut toutefois considérer que l'échantillon utilisé pour cette seconde recherche est beaucoup plus petit que celui employé en 1978 et qu'il est limité aux bibliothèques des collèges francophones de la région métropolitaine, ce qui ne permet pas de généraliser les résultats à l'ensemble du réseau des cégeps.

Dion, Gaudreau et Godin (1996) constatent pour leur part que la formation documentaire n'obtient pas la place qui devrait lui revenir dans l'enseignement collégial. Les auteurs trouvent regrettable que la formation documentaire ne soit «intégrée à aucun programme» et que la prestation des séances repose «sur l'initiative des responsables de bibliothèques et sur la bonne volonté des enseignants» (Dion, Gaudreau \& Godin 1996, 82). Le sondage qu'ils ont réalisé auprès des responsables des bibliothèques collégiales ( 23 répondants) les informe que, dans la majorité des cas, les "interventions se situent [...] au niveau d'un professeur et de ses groupes d'étudiants" plutôt qu'au niveau des départements lorsqu'il est question de "conce[voir] et [de] $m$ [ettre] en cuuvre d'activités d'apprentissage » en lien avec la forma- tion documentaire (Dion, Gaudreau \& Godin 1996, 83). Ceci empêche le bibliothécaire de faire valoir et de remplir pleinement son rôle de partenaire sur le plan pédagogique. Les auteurs espèrent que l'implantation de l'approche-programme au collégial, qui «vise à former des équipes éducatives [afin d']introduire plus de cohérence dans les interventions " faites auprès des étudiants, va inciter les bibliothécaires à se joindre aux discussions, à prendre «la totalité de l'espace pédagogique qui leur revient " et à collaborer plus étroitement avec des groupes d'enseignants en vue de développer chez les étudiants une meilleure maîtrise de l'information (Dion, Gaudreau \& Godin 1996, 83-84).

La dernière étude portant sur la formation documentaire au collégial est celle réalisée en 2002 par Marquis. Cette enquête a pour objectif de "brosser un tableau global des activités de formation documentaire offertes [... par le personnel des bibliothèques des cégeps " (Marquis 2002, 171). Quarante-quatre cégeps et collèges remplissent le questionnaire Web envoyé par Marquis. Les résultats obtenus viennent appuyer les constats faits par Pagé et Reid près de 15 ans plus tôt, à savoir que les "responsables de bibliothèques du réseau collégial reconnaissent l'importance de la formation documentaire ", puisque la totalité des répondants offre des activités et des services destinés à améliorer les compétences en recherche des étudiants (Marquis 2002, 172). Marquis s'intéresse particulièrement aux ateliers de formation donnés en laboratoire. Il souligne que ces séances sont majoritairement offertes dans des locaux spécifiquement aménagés à ces fins et estime que « $40 \%$ de la clientèle étudiante [est rejointe] par ce type de formation " pendant sa première année d'étude au cycle collégial (Marquis 2002, 172). En tout, Marquis rapporte que 1312 ateliers ont été offerts en 2001-2002 et qu'ils ont rejoint 31723 étudiants. D’après les données récoltées, "l'identification $d u$ besoin d'un atelier provient autant des enseignants que des responsables de bibliothèques" (Marquis 2002, 172). L'auteur note finalement que le nombre de professionnels en bibliothèque a un impact sur le pourcentage d'étudiants ayant la possibilité d'assister à un atelier. Il illustre cette découverte à l'aide de trois exemples :

Un cégep de 1400 étudiants pouvant compter sur un bibliothécaire professionnel parvient à rejoindre près de $85 \%$ de sa clientèle; un cégep de 3 ooo étudiants, desservi par deux bibliothécaires atteint un peu plus de $25 \%$ de sa clientèle, alors qu'un cégep de 5217 étudiants mais ne comptant qu'un seul professionnel ne peut atteindre que $7 \%$ de sa clientèle.

(Marquis 2002, 171-172)

Marquis conclut en proposant que la formation documentaire a une incidence "dans la réussite d'un programme collégial et [...] que celle-ci doit être offerte 
par le personnel professionnel ou technique de la bibliothèque " (Marquis 2002, 172).

\section{Méthodologie}

Après avoir pris connaissance des résultats de ces études, les membres du comité ont dû convenir d'une définition de la formation documentaire et des différentes formes qu'elle pouvait prendre. Cette définition a été largement éclairée par la pratique. Tel que noté par Turner, Dufour, Laplante, Leroux et Salaün, «les normes de l'Association of College and Research Libraries (ACRL) servent aujourd'hui en Amérique du Nord de cadre de référence pour la création de programmes de formation documentaire» $(2009,181)$. Pour les besoins de l'étude, la formation documentaire a donc été définie comme toute initiative, développée par la bibliothèque d'un collège ou d'un cégep, permettant à l'étudiant d'améliorer ses compétences informationnelles (CI), en adéquation avec les standards identifiés en 2000 par l'ACRL. Il est à noter qu'au moment de conduire l'enquête, le nouveau Framework for Information Literacy for Higher Education (ACRL 2015) n'était pas encore connu. Nous nous sommes donc inspirés des Information Literacy Competency Standards for Higher Education (ACRL 2000) et de leur traduction française, telle que réalisée par le Groupe de travail sur la formation documentaire du Sous-comité des bibliothèques de la Conférence des recteurs et des principaux des universités du Québec (CREPUQ 2005). Les cinq standards de CI, relatifs aux aptitudes des étudiants, sont les suivants :

1. L'étudiant est capable de déterminer la nature et l'étendue de l'information dont il a besoin;

2. L'étudiant accède avec efficacité et efficience à l'information dont il a besoin;

3. L'étudiant évalue de façon critique l'information et les sources trouvées. Il intègre l'information sélectionnée à sa base de connaissances personnelle et à son système de valeurs;

4. L'étudiant utilise efficacement l'information, que ce soit individuellement ou comme membre d'un groupe, en vue d'atteindre un objectif spécifique;

5. L'étudiant comprend plusieurs des questions économiques, juridiques et sociales relatives à l'utilisation de l'information. Il accède à l'information et l'utilise d'une manière éthique et conforme à la loi ${ }^{1}$.

En se basant sur cette définition et sur ces standards de compétences informationnelles, le comité a convenu que les ateliers de formation en classe, les ateliers en laboratoire informatique, les visites de la bibliothèque, les activités de type rallye, chasse au trésor ou jeu de piste en bibliothèque ou sur Internet, les guides d'initia-

1. Standards adaptés d'ARCL (2000) et de CREPUQ (2005). tion ou d'information, les pages thématiques ou les capsules sur le site de la bibliothèque, les tutoriels, de même que les formations individuelles (aide à la recherche sur place ou à distance) étaient tous considérés comme des formes de formation documentaire au regard de la présente étude.

Le comité a ensuite entrepris l'élaboration du questionnaire de recherche en se basant en partie sur celui administré par Marquis en 2002. Le questionnaire utilisé par Marquis (2002), composé de 10 sections, compte plusieurs questions d'opinion, visant davantage à évaluer le degré d'importance accordé par les responsables de bibliothèque à la formation documentaire qu'à recenser les pratiques. Par ailleurs, pour Marquis, la formation documentaire se définit comme "l'ensemble des activités de formation offertes par le personnel d'une bibliothèque, visant l'appropriation des compétences de base inhérentes à la recherche dans les documents imprimés ou en ligne» $(2002,169)$, définition qui n'inclut pas l'évaluation des sources repérées (standard 3), l'emploi des documents trouvés en vue de réaliser un projet (standard 4) et l'utilisation éthique de l'information (standard 5). Aussi, les environnements informationnel et pédagogique ayant passablement évolué entre le début du millénaire et le présent, le comité a alors entièrement retravaillé le questionnaire, ajouté des questions prenant en compte une définition plus large de la formation documentaire et fait en sorte que des données quantitatives complétant les données qualitatives puissent également être comptabilisées.

Le nouveau questionnaire comporte 24 éléments : une zone d'identification, 18 questions à choix de réponses et trois questions à court développement (17 de ces questions étaient complétées par une zone permettant l'ajout d'un autre choix de réponse, d'une précision ou d'un commentaire relatif à cette question), une question à long développement et une boîte de commentaires généraux. Les questions ont trait à la conception et à la prestation des formations documentaires, aux catégories d'emploi des personnes participant au processus de formation, aux équipements utilisés, à la durée des formations, aux types de formations, au nombre d'ateliers offerts et d'étudiants y assistant, aux contenus présentés, aux évaluations effectuées à la suite d'un atelier de formation, à l'intégration des formations dans le cursus, ainsi qu'aux styles bibliographiques supportés.

Le questionnaire est réalisé en ligne grâce à l'outil Survey Monkey. En mars 2014, il est acheminé à six bibliothécaires et spécialistes en moyens et techniques d'enseignement $(\mathrm{SMTE})^{2}$ à titre de pré-test. Le pré-test permet de valider globalement le questionnaire. Des modifications sont toutefois apportées afin de clarifier certaines questions, d'ajouter des éléments visant une

2. SMTE est le titre que portent la plupart des professionnels œuvrant en bibliothèque collégiale. Afin de ne pas alourdir le texte, seul le terme " bibliothécaire " sera utilisé dans la suite de cet article. 
meilleure mise en contexte, d'augmenter le nombre de choix de réponses à certains endroits, de donner des exemples ou de les retrancher. Une fois remanié, le questionnaire est envoyé à l'ensemble des responsables de la formation documentaire des collèges et cégeps membres du REBICQ via la liste de diffusion de l'organisation. La collecte des données a lieu entre le 9 et le 25 avril 2014. Quarante-trois répondants, sur une possibilité de 53, complètent le questionnaire, ce qui constitue un taux de réponse de $81 \%$. Étant donné ce taux élevé de répondants, nous sommes d'avis que les résultats obtenus sont représentatifs de la population à l'étude. Par ailleurs, comme le note Marquis dans son article sur la formation documentaire, ce fort taux de réponse « témoign[e] de l'intérêt de la question chez les responsables de bibliothèques collégiales " $(2002,171)$.

\section{Description de l'échantillon}

Il convient de relever une différence entre les répondants de l'étude de 2002 et celle de 2014. Marquis a sondé les responsables de bibliothèque (généralement des cadres ou des professionnels), alors que le comité a demandé à ce que les responsables de la formation documentaire (principalement des professionnels ou techniciens) remplissent le questionnaire. L'étude du comité cible donc les praticiens plutôt que les gestionnaires. Sur la population sondée, 34 sont des professionnels,

\section{Figure 1}

Pourcentage de bibliothécaires, de techniciens et d'enseignants responsables de la conception des formations

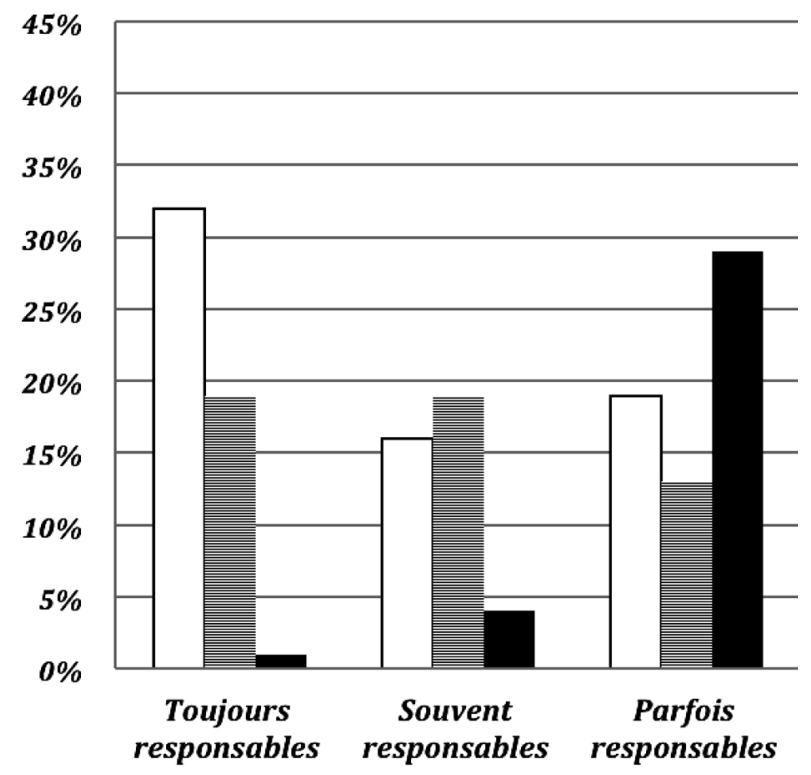

cinq sont des employés de soutien et quatre sont des cadres. Quarante d'entre eux œuvrent dans une institution publique, alors que trois travaillent dans un collège privé. Trente-neuf sont issus d'un établissement francophone, quatre proviennent d'un collège anglophone.

\section{Résultats}

L'enquête a permis de vérifier que la formation documentaire, sous toutes ses formes, est une pratique bien établie au collégial. En effet, $100 \%$ (43/43) des répondants ont affirmé offrir de la formation documentaire et $95 \%(41 / 43)$ d'entre eux proposent des ateliers en présentiel (ayant lieu en classe ou en laboratoire informatique). En règle générale, la conception et la prestation des formations sont confiées aux bibliothécaires, avec un apport significatif des techniciens en documentation et une participation occasionnelle des professeurs. Les figures 1 et 2 montrent cette répartition des rôles.

Le choix et l'utilisation des outils de recherche, l'exploration du site Web de la bibliothèque, l'identification des concepts et la sélection des mots clés, l'évaluation des sources, les fonctionnalités de recherche des outils, la distinction entre les différents types de documents, de même que la sensibilisation au plagiat sont les éléments les plus souvent abordés en formation. Le détail des contenus proposés est présenté au tableau 1.

\section{Figure 2}

Pourcentage de bibliothécaires, de techniciens et d'enseignants responsables de la prestation des formations

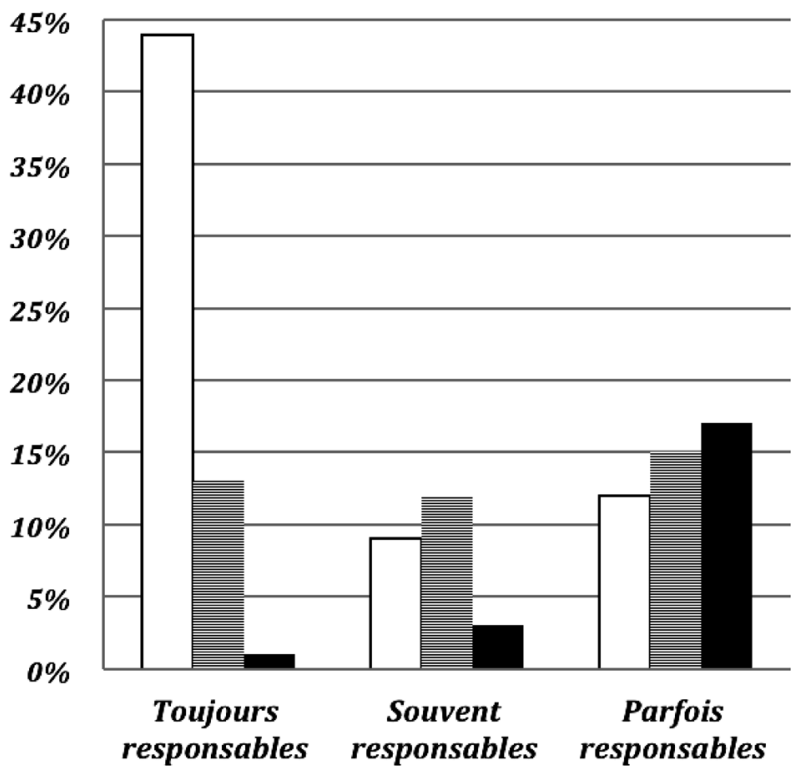


Tableau 1

Contenus abordés lors des formations offertes dans les bibliothèques collégiales, classés selon leur fréquence

\begin{tabular}{|c|c|}
\hline Contenus & $\begin{array}{l}\text { Pourcentage } \\
\text { de bibliothèques } \\
\text { abordant } \\
\text { cet élément } \\
\text { en formation }\end{array}$ \\
\hline Choix des outils de recherche & $98 \%$ \\
\hline Exploration du site Web de la bibliothèque & $93 \%$ \\
\hline Identification des mots clés pertinents & $88 \%$ \\
\hline $\begin{array}{l}\text { Évaluation de la qualité ou de la pertinence } \\
\text { des sources }\end{array}$ & $86 \%$ \\
\hline $\begin{array}{l}\text { Utilisation des fonctionnalités des outils } \\
\text { de recherche }\end{array}$ & $84 \%$ \\
\hline Distinction des divers types de documents & $81 \%$ \\
\hline Sensibilisation au plagiat & $81 \%$ \\
\hline $\begin{array}{l}\text { Survol de l'information générale } \\
\text { sur la bibliothèque }\end{array}$ & $77 \%$ \\
\hline Utilisation des opérateurs booléens & $74 \%$ \\
\hline Identification (avec précision) du sujet & $72 \%$ \\
\hline $\begin{array}{l}\text { Citation des sources et rédaction } \\
\text { d'une bibliographie }\end{array}$ & $67 \%$ \\
\hline Définition du besoin d'information & $63 \%$ \\
\hline Rédaction d'une équation de recherche & $56 \%$ \\
\hline Utilisation des livres numériques & $30 \%$ \\
\hline $\begin{array}{l}\text { Utilisation d'un logiciel de gestion } \\
\text { bibliographique }\end{array}$ & $9 \%$ \\
\hline Autre & $5 \%$ \\
\hline
\end{tabular}

La plupart des ateliers dispensés en classe ou en laboratoire ont une durée qui varie de 31 à 60 minutes $(67 \%$ des cas), $31 \%$ des séances demandent plus d'une heure et $2 \%$ durent moins de 30 minutes. Par ailleurs, les ateliers de formations s'insèrent dans une variété de cours et de programmes, à la fois préuniversitaires et techniques. Des étudiants de domaines aussi divers que le sont, par exemple, les sciences de la nature, l'éducation à l'enfance, la comptabilité et la gestion, les soins infirmiers, le design industriel, la diététique, la réadaptation physique, le travail social, la santé animale, la logistique du transport ou l'hygiène dentaire se voient ainsi offrir des ateliers. En tout, nous évaluons que $38 \%$ des étudiants inscrits à l'enseignement régulier ont assisté à un atelier au cours de l'année scolaire 2013-2014. Cette estimation est basée sur le nombre total d'étudiants rencontrés lors d'une séance de formation en présentiel (46 280) divisé par l'effectif étudiant en termes d'équivalent temps complet (ETC) des collèges (120 715), tels que déclarés par les répondants.

Si ces résultats font montre, dans l'ensemble, d'une image positive des formations documentaires en milieu collégial, l'étude a également mis en lumière certains aspects moins favorables. Notons d'abord que, dans la vaste majorité des cas, les ateliers de formation offerts par le personnel des bibliothèques sont organisés sur une base ponctuelle, à la suite d'une requête d'un professeur. Seuls sept répondants (16\%) ont affirmé que ces ateliers étaient prévus dans les plans-cadres des cours. Lorsque cette mesure n'est pas en place, cela signifie souvent que seuls les professeurs déjà conscients de la valeur des formations choisissent de réserver une séance pour leurs étudiants. À défaut d'une approche plus structurée, institutionnalisée, les bibliothécaires et techniciens ne sont pas en mesure de rejoindre la majorité de leur clientèle étudiante par des formations en présentiel. Par ailleurs, les résultats de l'enquête indiquent que plus le nombre d'ETC d'un cégep ou d'un collège est élevé, plus le pourcentage d'étudiants formés lors d'ateliers décroit.

Dans ce nuage de points, le nombre d'ETC est mis en relation avec le pourcentage d'étudiants rejoints par les ateliers (coefficient de corrélation : -0,29; corrélation moyenne négative ${ }^{3}$ ). Ceci suggère que plus l'institution d'enseignement est grande, moins nombreux (en proportion) sont les étudiants formés.

Une formalisation de l'offre de formation permettrait, selon les données recueillies, d'augmenter le pourcentage d'étudiants profitant d'ateliers en classe ou en laboratoire. En effet, il a été observé que, lorsque la tenue des ateliers est convenue avec le département, la discipline, le comitéprogramme ou la direction des études, plutôt qu'avec un professeur ou qu'à l'initiative de la bibliothèque seulement, le pourcentage d'étudiants rejoints augmente significativement (46\%par rapport à $31 \%)$.

L'accroissement du nombre de bibliothécaires dans chaque institution aurait également un impact positif sur le pourcentage d'étudiants formés. Dans le graphique qui suit (figure 4), la corrélation entre le nombre de bibliothécaires par ETC et le nombre d'ateliers de formation offerts apparaît clairement.

Il est vrai que les bibliothécaires ne sont pas les seuls responsables des formations documentaires offertes en bibliothèque collégiale. Comme mentionné plus tôt, les techniciens en documentation participent de manière significative à l'élaboration et à la prestation des formations. Il demeure toutefois que l'essentiel des activités de formation est conçu et animé par les professionnels et qu'une corrélation forte (coefficient de 0,58 ) existe entre le ratio de professionnels en bibliothèque par ETC et le nombre d'ateliers proposés aux étudiants.

Un autre facteur paraît avoir de l'importance lorsqu'il s'agit d'offrir des ateliers : la disponibilité d'un local de formation à l'intérieur même de la bibliothèque. Près de $42 \%(18 / 43)$ des répondants ont déploré de n'avoir pas accès à une telle salle. Une bibliothécaire formulait d'ailleurs ce commentaire à propos de l'absence de local de formation : "Malheureusement... J'aimerais mieux un espace adapté dans la bibliothèque, mais je dois plutôt me déplacer en classe où il y a parfois des ordinateurs et parfois non. " Lorsqu'ils ne disposent pas d'un local, les formateurs doivent réserver un laboratoire ou se rendre en salle de classe, ce qui complique la presta-

3. L'analyse de la force des corrélations est basée sur les barèmes établis par Cohen (1992): «En sciences humaines, on considère qu'une corrélation est forte à partir de ,50 environ, qu'elle est moyenne aux alentours de ,30, et qu'elle est faible vers, 10 » (Cohen, cité dans Fiske 2008, p. 79). 
Figure 3

Pourcentage d'étudiants rejoints selon la taille des cégeps répondants (en ETC)

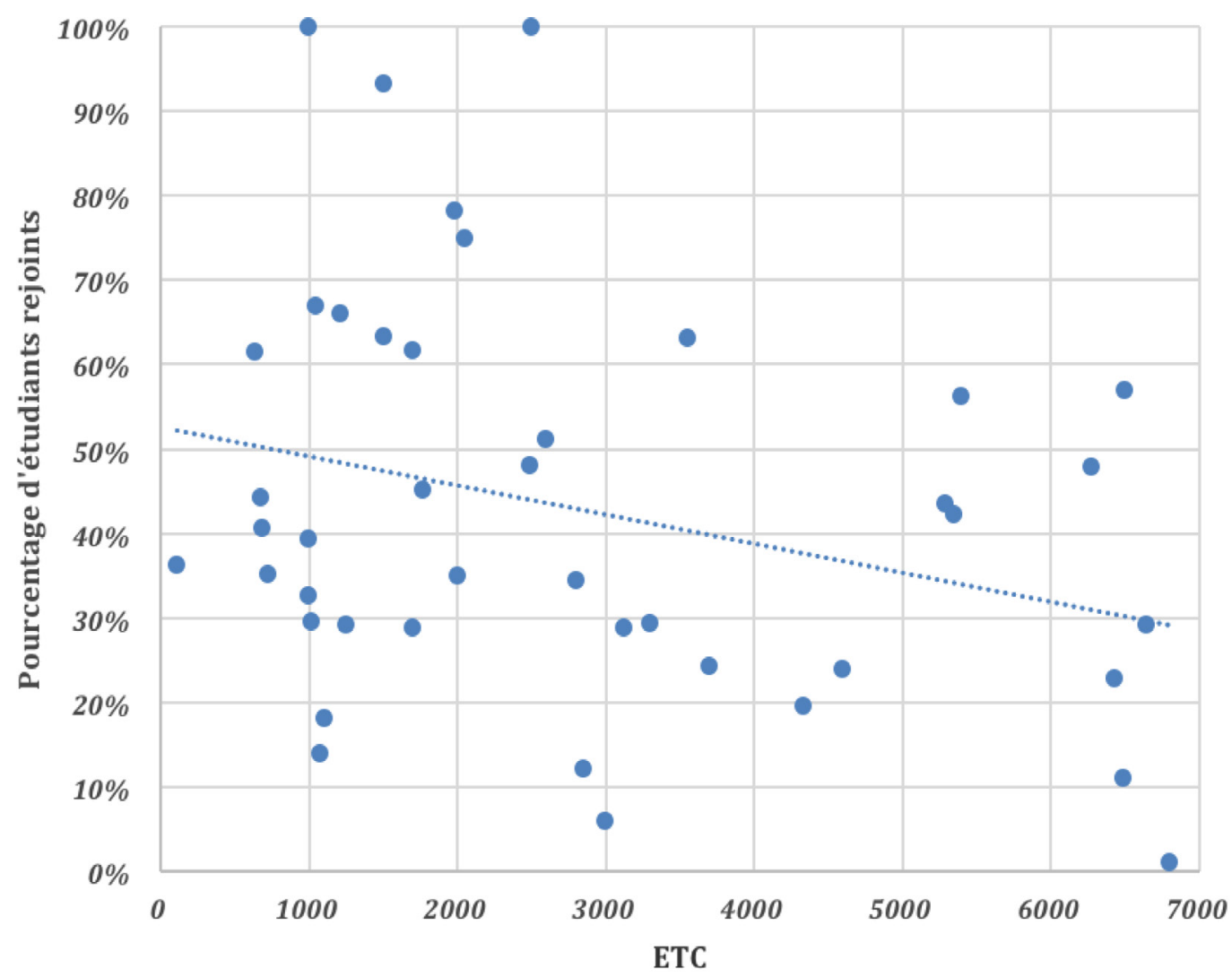

Figure 4

Nombre d'ateliers de formation donnés en fonction du ratio personnel professionnel/ETC

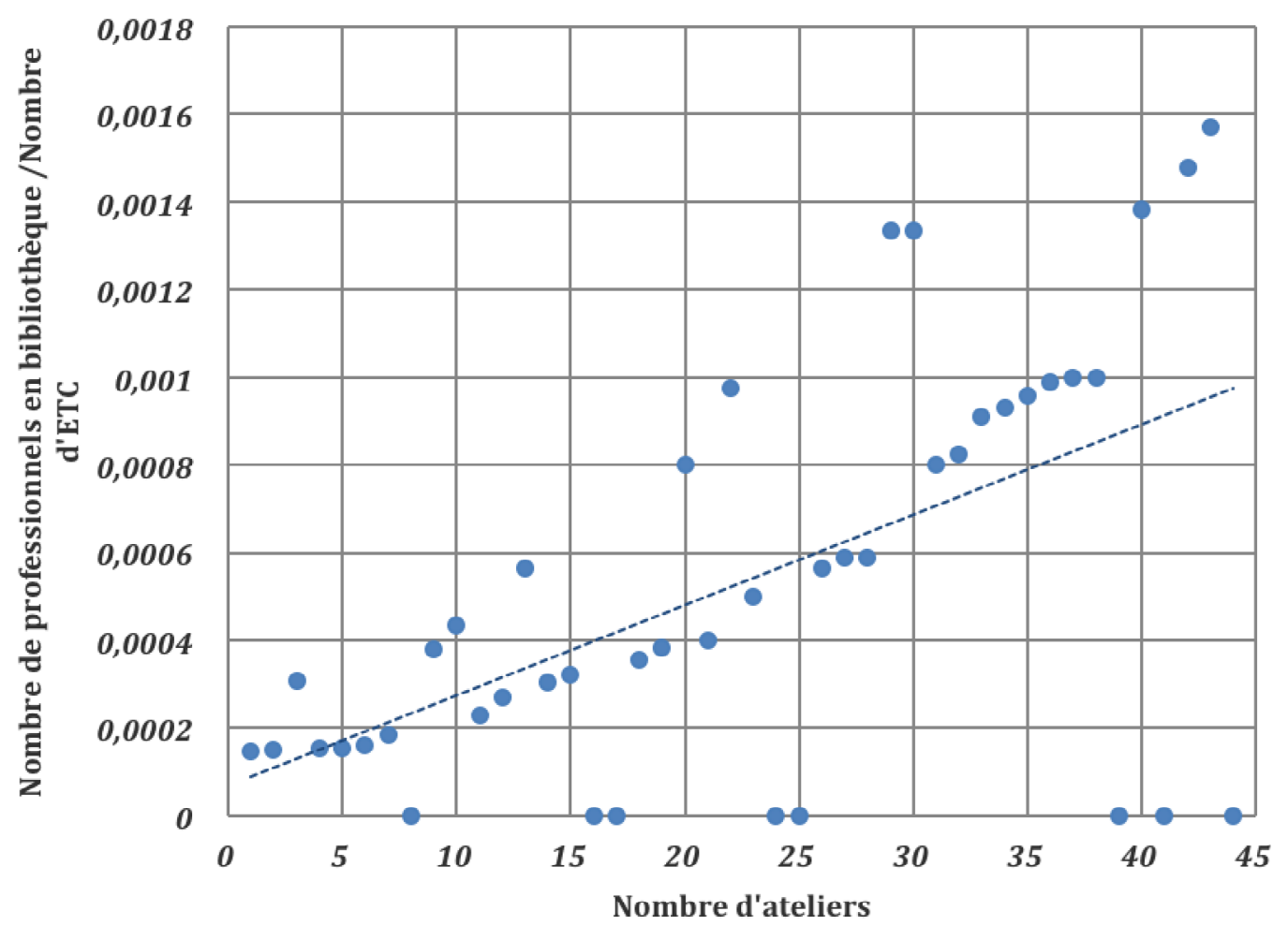

38 | JANVIER • MARS 2016 | DOCUMENTATION ET BIBLIOTHÈQUES 
tion des formations (manque de disponibilité de locaux appropriés, impossibilité pour les étudiants d'effectuer des exercices dans les bases de données, changement de la formule de formation, etc.).

Des lacunes se font sentir lorsqu'il est question des évaluations relatives aux formations. Moins de $20 \%$ des répondants (8/43) ont indiqué que les étudiants étaient évalués directement à la suite d'un atelier, d'une manière formative ou sommative. En l'absence de ces évaluations, il est difficile de mesurer les effets des formations sur le développement des compétences informationnelles chez les participants. On constate également que seul un peu plus du quart des répondants $(26 \%, 11 / 43)$ a mis en place des mesures pour faire évaluer l'offre de formation documentaire. Il s'agit habituellement d'un sondage distribué aux étudiants à la suite d'un atelier pour leur demander leur appréciation. Ainsi, l'évaluation des formations par les participants demeure une pratique peu répandue. Nous sommes certainement en présence d'une faiblesse, car sans rétroaction, il est plus difficile pour les formateurs de s'engager dans une pratique réflexive, puis d'apporter des améliorations de natures pédagogique ou technique aux séances offertes.

\section{Discussion}

Dans leur ensemble, les résultats de l'enquête conduite par le comité sur la formation documentaire du REBICQ viennent corroborer ceux obtenus lors des études précédentes, tout en apportant des précisions et en actualisant les données. Tel qu'observé par Pagé et Reid (1988), puis par Marquis (2002), la formation documentaire revêt une grande importance dans les bibliothèques collégiales puisque $100 \%$ des répondants en proposent. Toutefois, l'offre d'un atelier de formation à un groupe d'étudiants dépend encore, la plupart du temps, de la volonté d'un enseignant, non de la direction des études ou de la coordination de programme, une situation déplorée dès 1996 par Dion, Gaudreau et Godin, qui perdure en 2002 et qui est toujours le cas de figure le plus fréquent en 2014. Par ailleurs, la corrélation positive entrevue par Marquis (2002) entre le ratio de professionnels en bibliothèque par ETC et le nombre d'ateliers dispensés a été validée et il a été établi qu'elle était forte.

Il est aussi à noter que les contenus des formations ont évolué. Les responsables des formations documentaires en bibliothèque collégiales ne se contentent plus seulement d'aborder des éléments relevant de l'orientation ou la méthodologie de recherche. En sensibilisant les étudiants à la définition de leur besoin d'information, à l'utilisation des fonctionnalités des outils de recherche, à l'évaluation de la qualité et de la pertinence des sources, ainsi qu'au plagiat, les bibliothécaires et techniciens contribuent au développement des CI chez les cégépiens au regard des cinq standards reconnus par l'ACRL (2000).
L'analyse des données de l'enquête permet d'identifier plusieurs pistes d'action qui pourraient avoir une incidence bénéfique sur l'offre de formation documentaire au collégial. D’abord, il semble nécessaire que les différents acteurs appelés à soutenir l'apprentissage des étudiants (professeurs, conseillers pédagogiques, bibliothécaires, etc.) collaborent davantage afin que les formations aux CI offertes en bibliothèque soient mieux connues et plus reconnues au sein des institutions d'enseignement. En travaillant ensemble de manière plus concertée, ces intervenants pourraient faciliter une intégration plus formelle des formations documentaires au cursus des étudiants (inscription dans les plans-cadres; sollicitation des formations par le département, la discipline, le comité-programme ou la direction des études). Dans le cas où la systématisation des formations ne serait pas réalisable, il serait toujours possible pour les bibliothécaires de vérifier quels éléments de compétence inscrits dans les plans-cadres sont en lien avec les CI, ce qui leur permettrait de suggérer aux professeurs dispensant les cours visés qu'une formation soit offerte à leurs étudiants au moment opportun.

En deuxième lieu, nous ne pouvons qu'espérer voir augmenter le nombre de professionnels en bibliothèque dans les cégeps et collèges. Non seulement peut-on raisonnablement présumer que cet accroissement aura un impact positif sur le nombre d'ateliers de formation offerts (voir la figure 4), mais on peut également émettre l'hypothèse que l'embauche de bibliothécaires supplémentaires dans les établissements de grande taille permettrait de redresser la courbe tendance présentée à la figure 3 et ferait en sorte qu'un pourcentage plus élevé d'étudiants soient formés aux CI. Par ailleurs, il serait souhaitable que les formateurs (et les participants) bénéficient de conditions adéquates lors de la tenue des ateliers, notamment en ayant un local de formation à disposition à l'intérieur de la bibliothèque.

\section{Conclusion et ouverture}

L'enquête réalisée par le comité sur la formation documentaire a permis d'obtenir des données sur les pratiques actuelles de formation aux compétences informationnelles en milieu collégial. Plusieurs éléments positifs ont été mis en lumière par l'étude : tous les répondants ont affirmé offrir de la formation sous une forme ou une autre, les contenus des formations sont diversifiés et pertinents, les ateliers en classe ou en laboratoire sont dispensés dans une grande variété de cours et de programmes, les formateurs ont eu l'occasion de rencontrer en atelier plus du tiers des étudiants inscrits à l'enseignement régulier en 2013-2014. Le personnel des bibliothèques collégiales (bibliothécaires et techniciens) est donc déjà très actif dans le domaine des formations documentaires. Pour que la formation rejoigne encore plus d'étudiants, il faudra vraisemblablement octroyer plus de ressources (augmentation du nombre 
de bibliothécaires dans les institutions; aménagement de salles de formation dans les bibliothèques) et formaliser l'offre d'ateliers (inscription dans les plans-cadres; accord avec le département, la discipline, le comité-programme ou la direction des études). Il serait également souhaitable pour les formateurs de remédier à certaines faiblesses, notamment en ce qui a trait à l'évaluation des formations.

Cette étude est l'amorce d'un projet plus vaste pour le comité sur la formation documentaire. Maintenant que le portrait de l'offre de formation au collégial a pu être tracé, le comité s'attachera à la question de la réussite étudiante en lien avec les compétences informationnelles. Une revue de littérature, pilotée en collaboration avec deux professeurs de l'EBSI, est en cours. Le comité espère que cette deuxième phase de la recherche révèlera des façons d'évaluer l'impact du développement des CI sur le parcours académique des étudiants.

\section{Contributions}

L'auteure souligne la contribution des personnes suivantes à la rédaction de cet article :

Andrée Dagenais, bibliothécaire professionnelle, Centre de documentation collégiale

Lorrie Jean-Louis, animatrice du REBICQ

Isabelle Laplante, bibliothécaire professionnelle, Centre de documentation collégiale

Julie Larocque, bibliothécaire professionnelle, Cégep de St-Laurent

Philippe Lavigueur, spécialiste en moyens et techniques d'enseignement, Collège Montmorency

Daniel Marquis, Conseiller pédagogique et bibliothécaire professionnel, Cégep de Granby

Josiane Sauvé, spécialiste en moyens et techniques d'enseignement, Collège Lionel-Groulx

Les personnes suivantes ont également contribué aux travaux du comité sur la formation documentaire :

Nancie Lamontagne, M. S. I.

Alexandra Lavallée, M. S. I.

Nathalie Ouellet, M. S. I.

Une liste de contributeurs a été ajoutée dans cet article. Par conséquent, celui-ci diffère de la version imprimée.

\section{Sources consultées}

ACRL. (2000). Information Literacy Competency Standards for Higher Education. <http://www.ala.org/acrl/standards/ informationliteracycompetency> (consulté le 26 mai 2015).

ACRL. (2015). Framework for Information Literacy for Higher Education. <http://www.ala.org/acrl/standards/ilframework> (consulté le 26 mai 2015).

CREPUQ. (2005). Norme sur les compétences informationnelles dans l'enseignement supérieur de l'Association of College and Research Libraries. <http://www.crepuq.qc.ca/IMG/pdf/ normeacrl-web-03-05-v4.pdf> (consulté le 26 mai 2015).

Dion, H., Gaudreau, L., \& Godin, M. (1996). La maîtrise de l'information: un défi pédagogique à partager. Documentation et bibliothèques, 42 (2), 81-85.

Fiske, S. T. (2008). Psychologie sociale. Bruxelles: De Boeck.

Lemaire, J. (1978). L'apprentissage du travail personnel et de l'utilisation de la bibliothèque dans les Cégeps du Québec. Argus, 7 (2), 38-41.

Marquis, D. (2002). La formation documentaire dans les bibliothèques collégiales: état de la question et enquête. Documentation et bibliothèques, 48 (4), 169-172.

Pagé, L. \& Reid, J. (1988). La formation documentaire dans les collèges de la région de Montréal. Documentation et bibliothèques, 34 (4), 133-141.

REBICQ. (2015a). Mission du Regroupement des bibliothèques collégiales du Québec. <http://www.rebicq.ca/qui-sommesnous/mission-du-regroupement-des-bibliotheques-collegialesdu-quebec/> (consulté le 26 mai 2015).

REBICQ. (2015b). Nos comités. <http://www.rebicq.ca/qui-sommesnous/nos-comites/> (consulté le 26 mai 2015).

Turner, J., Dufour, C., Laplante, A., Leroux, E., \& Salaün, J.-M. (2009). Les pratiques des utilisateurs. Dans J.-M. Salaün, \& C. Arsenault (dir.), Introduction aux sciences de l'information (p. 159-182). Montréal : Presses de l'Université de Montréal. 